\title{
INGÉNIERIE DES MATÉRIAUX SEMI-CONDUCTEURS: \\ le cas des structures à barrière pour la photodétection infrarouge
}

Philippe CHRISTOL

Université de Montpellier, IES, UMR 5214,

34000 Montpellier, France

CNRS, IES, UMR 5214,

34000 Montpellier, France

*philippe.christol@umontpellier.fr
Les matériaux semiconducteurs III-V de la «famille» des antimoniures (III-Sb), c'est-à-dire les semiconducteurs GaSb, InAs, AlSb et leurs alliages, présentent des raccordements de bande interdite si particuliers qu'ils permettent la conception de structures très originales. C'est le cas des structures à barrière dites $X B n$ qui se sont imposées ces dernières années comme I'un des standards des dessins de photodétecteurs infrarouges haute performance refroidis. et performantes. Depuis cette date, un nouveau concept de composant s'est imposé pour la photodétection IR haute performance refroidie: le détecteur à barrière, appelé XBn ou bariode.

Dans l'objectif de réduire le courant d'obscurité, donc le bruit du composant, le premier détecteur IR à barrière fut proposé par White en 1983 [1]. Il proposait une architecture composée d'hétérostructures semiconductrices isotypes (de type n) où une couche barrière à grande énergie de bande interdite (gap) était placée entre les couches absorbante et contact de matériaux à plus petit gaps. Comme l'objectif de la couche barrière est de bloquer les porteurs majoritaires

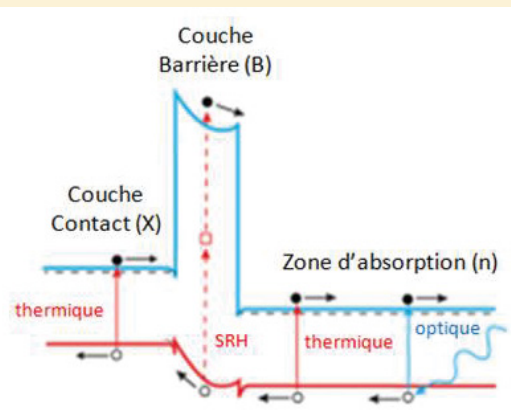

photodiode à jonction pn détecteur à barrière XBn
Figure 1. Diagramme de bande d'énergie d'une photodiode à jonction pn et d'un détecteur à barrière XBn. (ici les électrons) tout en laissant passer les porteurs minoritaires (trous) photogénérés, il appela son dispositif "photoconducteur à haute impédance». Cependant à l'époque, obtenir un offset de bande de valence très faible voire nul était difficile à réaliser avec les matériaux phares de la détection IR qu'étaient l'InSb et le HgCdTe. Tout changea au milieu des années 2000 avec la démonstration du détecteur nBn à InAs [2] puis s'accéléra avec les matériaux semiconducteurs III-Và base d'antimoniures $\mathrm{Sb})$ et la fabrication de couches alliages en InAsSb mais aussi en utilisant les propriétés d'ingénierie de bande interdite et $\mathrm{d}$ 'offset des structures à super-réseaux de type-II (T2SL) en accord de maille sur le substrat $\mathrm{GaSb}$.

\section{Pourquoi une structure à barrière XBn pour la photodétection IR?}

Comprendre les avantages du détecteur $\mathrm{XBn}, \mathrm{c}^{\prime}$ est avant tout comparer cette structure à barrière à la photodiode à jonction pn (figure 1). Dans la structure à jonction pn en polarisation inverse, la présence de la zone de charge d'espace de part et d'autre de l'interface métallurgique $\mathrm{p} / \mathrm{n}$ bloque le transport des 


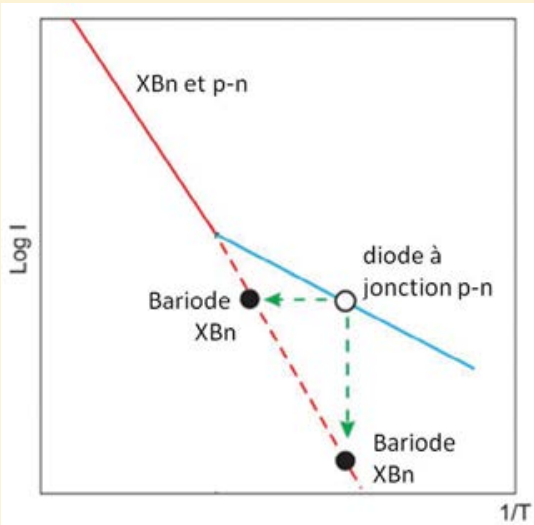

Figure 2. Représentation schématique d'un graphe d'Arrhenius traçant le courant d'obscurité en fonction de l'inverse de la température pour une diode à jonction pn et une bariode XBn.

porteurs majoritaires et autorise celui des porteurs minoritaires. Cependant, cette zone avec présence de champ électrique engendre un courant d'obscurité de recombinaison Shockley-Read-Hall (SRH) qui peut être important car les gaps des matériaux adressant l'IR sont faibles. De ce fait, le courant d'obscurité de la photodiode est dominé par ce courant SRH à basse température, et par le courant de diffusion des porteurs minoritaires vers la zone de champ électrique à plus haute température (figure 2). L'objectif premier de la structure XBn est de réduire précisément la contribution du courant SRH au courant d'obscurité du composant. Pour cela, il faut contrôler la zone de champ électrique en la confinant dans le matériau barrière à grand gap et cette barrière doit jouer le même rôle que la zone de charge d'espace dans la structure pn: bloquer les porteurs majoritaires (électrons sur la figure 1) et laisser passer les porteurs minoritaires (trous). La barrière XBn (voir encart 1) doit donc posséder un grand offset de bande de conduction et un faible offset de bande de valence pour ne pas entraver le signal (le photocourant). Si tel est le cas, la contribution du courant SRH au courant d'obscurité est fortement réduite et le composant XBn est dominé par le courant de diffusion quelle que soit la température (figure 2). Dès lors, vis à vis dela photodiode, la bariode XBn permet une réduction du courant d'obscuritéà iso-température, ou bien une augmentation de la température de fonctionnement à iso-performance.

\section{Ingénierie des matériaux pour les détecteurs IR à barrière}

Ces dernières années, les structures à barrière pour la photodétection IR se sont développées grâce aux propriétés particulières de la famille des matériaux semiconducteurs III-V au

\section{Les bariodes XBn et XBp}

XBn est un terme générique qui peut décliner toutes associations de matériaux semiconducteurs représentant une couche contact notée $X$, une barrière notée $B$ et une couche absorbante notée $n$. La couche barrière est le point clé de ce dispositif, d'où le nom de bariode. Elle doit bloquer les électrons majoritaires sans empêcher les trous photo-générés d'atteindre la couche contact. Le concept de structure à barrière peut aussi s'appliquer pour une zone d'absorption de type $p$. Dans ce cas la bariode s'appelle XBp et ce sont les trous majoritaires qui doivent être bloqués par une barrière de bande de valence, sans entraver, au niveau de la bande de conduction, le transport des porteurs minoritaires électrons. La figure suivante présente toutes les configurations possibles de structures XBn et XBp.

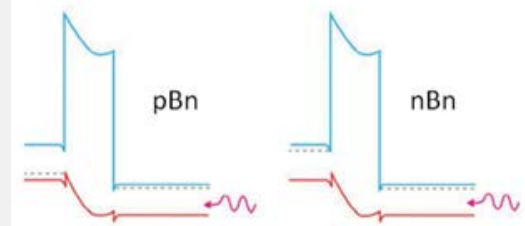

(a)

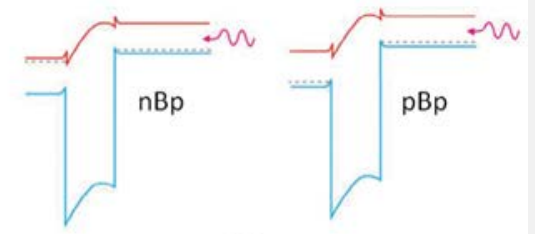

(b)

Représentation schématique des configurations possibles de structures XBn (a) et XBp (b). Dans chaque cas, la couche contact est à gauche et l'absorption du rayonnement IR s'effectue au niveau de la couche absorbante à droite (d'après [3]).

\section{IDIL SPÉCIALISTE DES TECHNOLOGIES FIBRES OPTIQUES ET LASER}

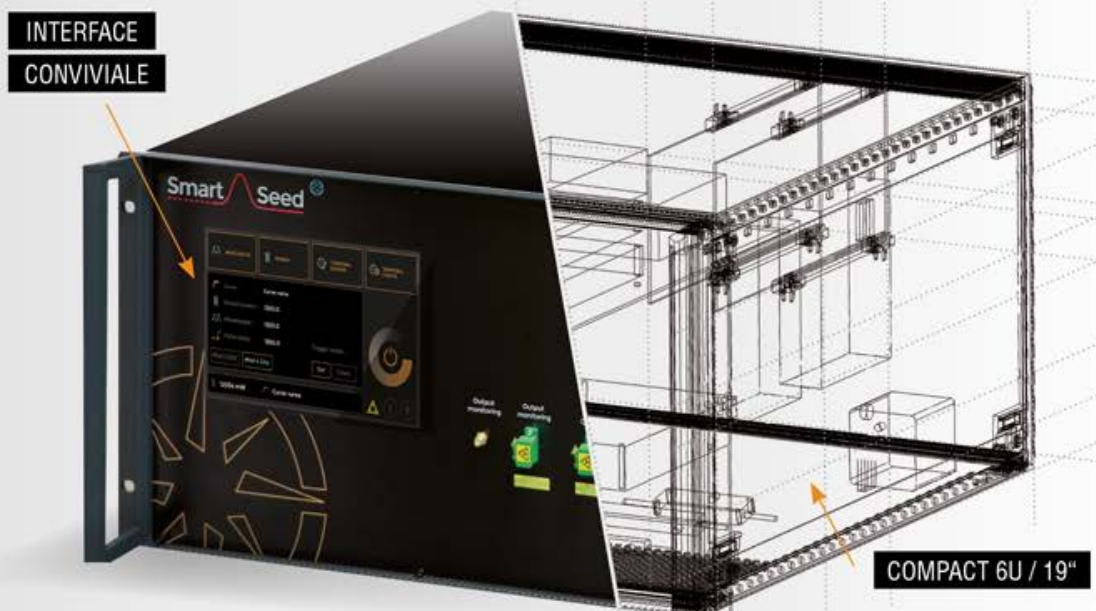

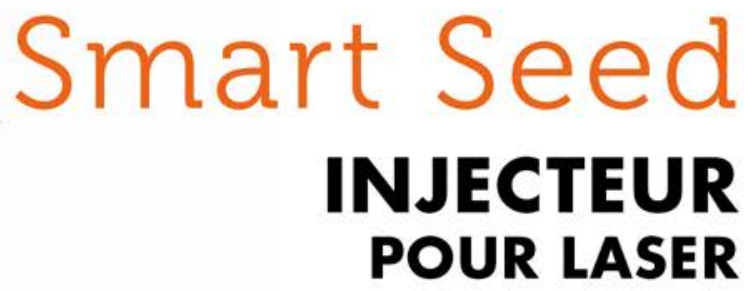

HAUTE PUISSANCE

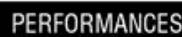

$\lambda 1030,1053,1060 \mathrm{~nm}+$ sur mesure Energie > $10 \mathrm{nj}$ ( $20 \mathrm{~ns}$ largeur d'impulsion)

Rapport d'extinction temporelle $>50 \mathrm{~dB}$ Forme d'impulsion ajustable

Résolution temporelle $125 \mathrm{ps}$

Durée d'impulsion 300 ps à $35 \mathrm{~ns}$

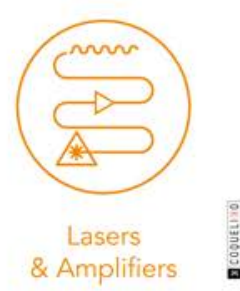




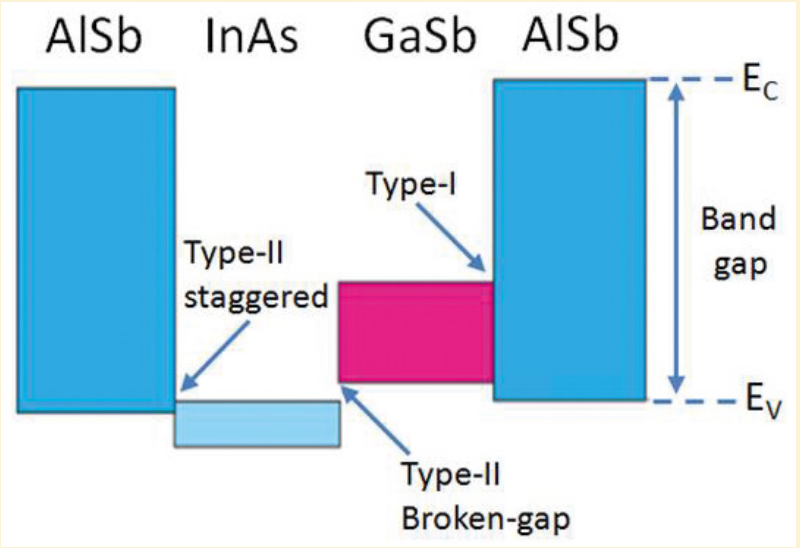

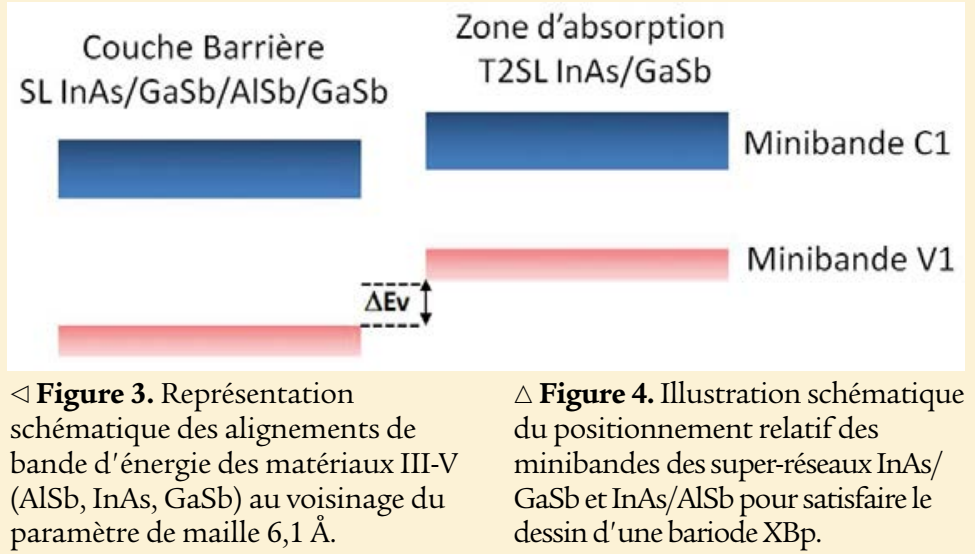

Zone d'absorption

T2SL InAs/GaSb

Minibande C1

Minibande V1 $\triangleleft$ Figure 3. Représentation schématique des alignements de (AlSb, InAs, GaSb) au voisinage du paramètre de maille 6,1 Å $\triangle$ Figure 4. Illustration schématique du positionnement relatif des GaSb et InAs/AlSb pour satisfaire le dessin d'une bariode XBp. voisinage du paramètre de maille $6,1 \AA$ du matériau $\mathrm{GaSb}$ (figure 3). C'est le cas du détecteur $\mathrm{XBn}$ InAsSb car il existe une barrière grand gap en AlAsSb accordée en maille au substrat $\mathrm{GaSb}$ qui présente les propriétés requises pour le transport des porteurs, en bloquant les électrons majoritaires et en collectant facilement sous faible polarisation les trous minoritaires. Des systèmes imageurs haute performance $\mathrm{XBn}$ InAsSb refroidis sont maintenant commercialement disponibles et affichent des températures de fonctionnement de $150 \mathrm{~K}$ pour la partie bleue du domaine spectral MWIR $(3,4-4,2 \mu \mathrm{m})$ [3]. Une telle température de fonctionnement permet une forte diminution du poids et de l'encombrement, du temps de mise en froid et de la consommation d'énergie de la machine à froid.

Un autre système de matériaux III-V utilise la configuration à barrière pour la photodection IR. Il s'agit du super-réseau de type-II (T2SL) InAs/ $\mathrm{GaSb}$ (voir encart 2). Toutefois, pour des raisons de propriétés de transport, les rendements quantiques des détecteurs T2SL InAs/GaSb sont optimisés pour des zones d'absorption de type $\mathrm{p}$. Par conséquent, les T2SL doivent être intégrés au sein de structures à barrière $\mathrm{XBP}$ (voir encart 1). Cependant, il n'existe pas de matériau alliage «naturel» présentant un offset de bande de valence qui permettrait de bloquer les trous sans entraver le déplacement des électrons. La seule solution est d'utiliser les propriétés de flexibilité des T2SL, associées aux matériaux de la famille $6,1 \AA$, afin de créer une barrière «artificielle» de trous par positionnement relatif des minibandes d'énergie des super-réseaux InAs/GaSb et InAs/AlSb (figure 4). Par ingénierie de la structure de bande, les périodes des super-réseaux sont choisies pour satisfaire la configuration XBp avec la création d'un offset de bande de valence $\Delta$ Ev suffisant pour bloquer les porteurs trous. On conçoit aisément que cette approche peut s'appliquer pour n'importe quel design de structures XBp ou XBn, MWIR ou LWIR. Mais la réalisation de ces super-structures périodiques complexes, sans atomes communs, demande des techniques et procédés de croissance sophistiqués, notamment par épitaxie

\section{Le T2SL}

Le T2SL, ou super-réseau de type-II, est un empilement de couches périodiques en InAs et en GaSb dont l'épaisseur est de l'ordre de quelques nanomètres, ce qui revient à considérer une structure quantique composée de multipuits quantiques couplés. De ce fait, les porteurs sont confinés dans des minibandes d'énergie en raison de la dégénérescence des niveaux quantifiées de tous les puits quantiques. Les transitions optiques s'effectuent donc ici non pas entre deux continuums d'énergie, comme dans le cas d'un matériau massif, ni entre deux niveaux discrets, comme dans le cas d'un puits quantique simple, mais entre deux de ces minibandes. Utilisée comme zone d'absorption d'un détecteur, cette structure quantique à l'interface très particulière de "type-II broken gap » (figure 3) présente une énergie de transition fondamentale (longueur d'onde de coupure du détecteur $\lambda_{c}$ ) dépendant uniquement des épaisseurs des deux binaires la constituant, avec une absorption interminibandes valence-conduction (V1-C1) pouvant adresser tout l'IR thermique, de 3 à $30 \mu \mathrm{m}$. C'est en partie grâce à sa grande flexibilité en longueur d'onde d'absorption que le T2SL fut proposé, à la fin des années 80 , comme matériau pour la détection IR [4].

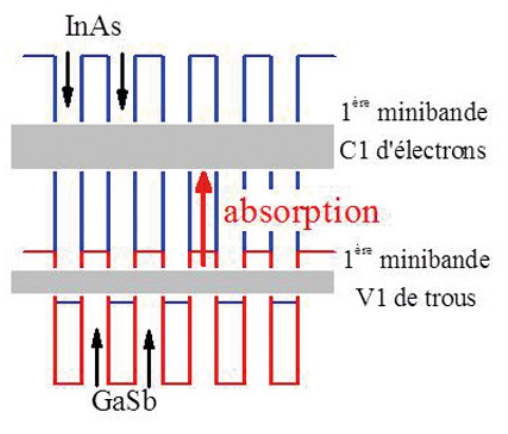

(a)

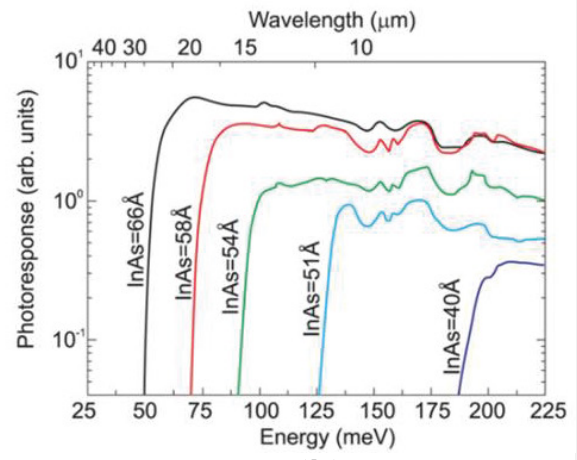

(b)
Représentation schématique de l'absorption interminibandes valence-conduction dans un super-réseau InAs/GaSb. Spectres de photoréponse à $80 \mathrm{~K}$ de photodétecteurs T2SL (d'après [5]). Les périodes des super-réseaux sont constituées de différentes épaisseurs d'InAs (de $40 \AA$ à $66 \AA$ ) associées à la même épaisseur de GaSb $(40 \AA$ ) $)$. 
par jets moléculaires, avec une gestion très précise des interfaces afin de contrôler l'accord de maille sur le substrat $\mathrm{GaSb}$.

En configuration XBp avec suppression des courants SRH, les T2SL sont alors très compétitifs vis à vis des autres filières de détecteurs établies, en particulier dans le LWIR. Depuis peu, de tels composants XBp T2SL sont commercialisés (figure 5), démontrant ainsi que cette filière de matériau a atteint une véritable maturité technologique dans le domaine de la détection IR.

\section{Conclusion}

Les matériaux semiconducteurs de la filière des antimoniures présentent des propriétés si singulières en termes de raccordement de bandes interdites, qu'ils stimulent l'imagination des concepteurs de composants optoélectroniques. Cette filière de matériaux permet la fabrication de nombreuses pièces du mécano quantique [6] par ingénierie de la structure de bande, que ce soit des zones actives de composants détecteurs inter ou intrabandes à base de super-réseaux de périodes simples, « $\mathrm{W} »$, « $\mathrm{M}$ » ou bien « $\mathrm{N}$ », ou bien des structures à grand gap ajustable à souhait pour composants à barrière XBn et XBp. Nous avons vu dans cet article que les structures à barrière, que l' on pourrait aussi appeler photoconducteur à porteurs minoritaires, ont permis ces dernières années une forte amélioration des performances des photodétecteurs T2SL MWIR et LWIR refroidis. Mais ce n'est qu'un début. Ce concept tend à se généraliser à d'autres filières de matériaux, comme par exemple les super-réseaux « Ga-free» InAs/InAsSb mais aussi le HgCdTe, pour couvrir tous les domaines spectraux de l'infrarouge, $y$ compris le très lointain infrarouge VLWIR $(\lambda>12 \mu \mathrm{m})$ pour applications spatiales.

\section{POUR EN SAVOIR PLUS}

[1] A. White, Infrared detectors, U.S. Patent 4679063 (22 septembre 1983)

[2] S. Maimon, G. Wicks, nBn detector, an infrared detector with reduced dark current and higher operating temperature, Applied Physics Letters 2006; 89: 151109.

[3] P.C. Klipstein, XBn and XBp infarred detectors, Journal of Crystal Growth 2015; $425: 351$.

[4] D.L. Smith, C. Mailhiot, Proposal for strained type II superlattice infrared detectors, Journal of Applied Physics $1987 ; 62: 2545$

[5] Y. Wei, M. Razeghi, Modeling of type-II InAs/GaSb superlattices using an empirical tight-binding method and interface engineering, Physical Review B 2004; 69 : 085316.

[6] E. Rosencher, Les puits quantiques et la détection infrarouge, La recherche 1992, 248: 1270.
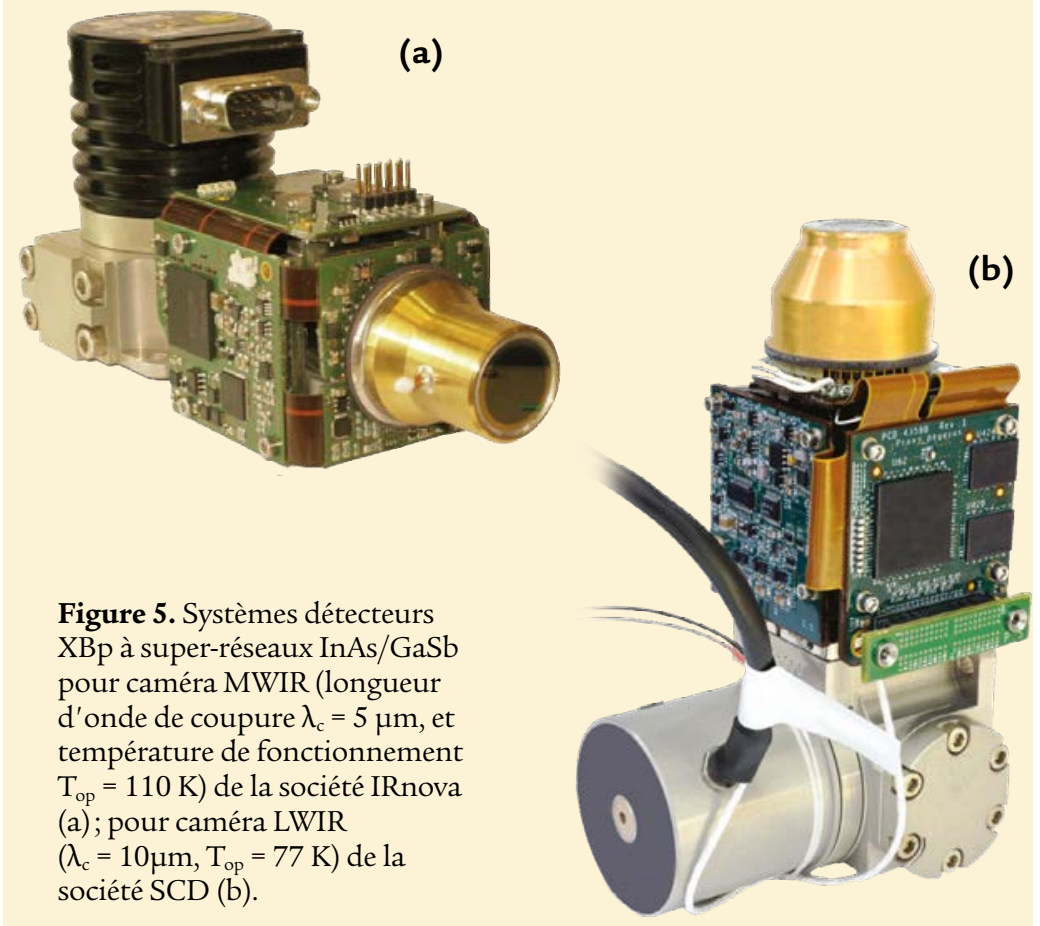

Figure 5. Systèmes détecteurs $\mathrm{XBp}$ à super-réseaux $\mathrm{InAs} / \mathrm{GaSb}$ pour caméra MWIR (longueur d'onde de coupure $\lambda_{\mathrm{c}}=5 \mu \mathrm{m}$, et température de fonctionnement $\mathrm{T}_{\mathrm{op}}=110 \mathrm{~K}$ ) de la société IRnova (a); pour caméra LWIR $\left(\lambda_{\mathrm{c}}=10 \mu \mathrm{m}, \mathrm{T}_{\mathrm{op}}=77 \mathrm{~K}\right)$ de la société $\mathrm{SCD}(\mathrm{b})$.

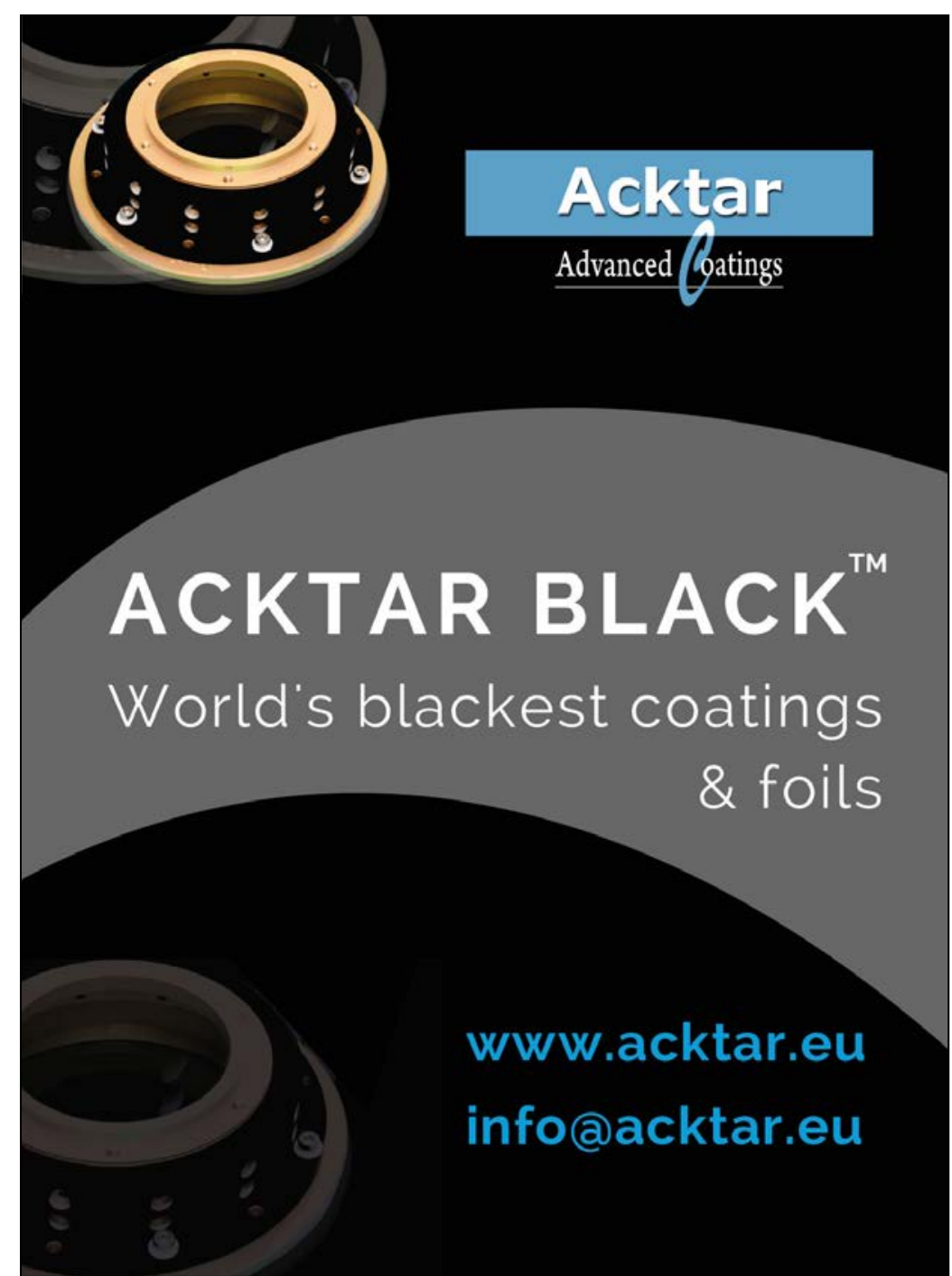

